\title{
The influence of low and moderate carotid stenosis on neurophysiologic status of patients undergoing on-pump coronary artery bypass grafting
}

\author{
Olga Trubnikova $^{1}$ *, Irina Tarasova ${ }^{2}$ and Olga Barbarash ${ }^{1}$ \\ Department of Multifocal Atherosclerosis, Research Institute for Complex Issues of Cardiovascular Diseases, Siberian Branch of the Russian Academy of Medical \\ Sciences, Kemerovo, Russian Federation \\ ${ }^{2}$ Department of Cardiovascular Diagnostics, Research Institute for Complex Issues of Cardiovascular Diseases, Siberian Branch of the Russian Academy of Medical \\ Sciences, Kemerovo, Russian Federation
}

\section{Edited by:}

Elena Pronina, Krasnoyarsk State

Medical University, Russia

\section{Reviewed by:}

Elena Pronina, Krasnoyarsk State

Medical University, Russia

Elena Mozheyko, Krasnoyarsk State

Medical University named after Prof.

V. F. Voyno-Yasenetsky, Russia

Alla B. Salmina, Krasnoyarsk State

Medical University named after Prof.

V. F. Voyno-Yasenetsky, Russia

\section{*Correspondence:}

Olga Trubnikova, Laboratory of

Neurovascular Pathology, Department

of Multifocal Atherosclerosis,

Research Institute for Complex Issues

of Cardiovascular Diseases, Siberian

Branch of the Russian Academy of

Medical Sciences, Boulevard

Sosnovy, 6, Kemerovo 650002,

Russian Federation.

e-mail:olgalet17@mail.ru
Background: Significant ( $>70 \%$ ) extracranial stenosis of the internal carotid artery (ICA) is a known risk factor for brain damage in patients with coronary heart disease (CHD) undergoing coronary artery bypass grafting (CABG). There is no clear evidence of the low and moderate ICA stenoses influence on the neurophysiologic status of patients after CABG. This work was aimed at studying the influence ICA stenoses $(<50 \%)$ on the dynamics of neurophysiologic status in patients undergone CABG. Methods: We examined neurophysiologic functions and electroencephalograph in CHD patients $(N=45)$ aged from 45 to 70 years. All patients were divided into two groups: with ICA stenosis $(n=20)$ and without one $(n=25)$. Results: It was established that the group ICA stenosis had a negative dynamics of neurophysiologic status 6 months follow-up after CABG compared with patients without stenosis. Conclusion: Our results suggest that the presence of low and moderate ICA stenosis is one of the factors affecting the neurophysiologic status of CHD patients. It has been assumed that the patients with $\leq 50 \%$ ICA stenoses constitute a high-risk group for cerebral complications after on-pump CABG.

Keywords: neurophysiologic status, cognitive impairment, on-pump CABG, EEG

\section{INTRODUCTION}

In the last decade one of the most discussable issues are the cerebral complications after coronary artery bypass grafting (CABG) due to its high social importance and the data ambiguity. Severe cerebral complications after cardiac surgery, including a general cerebral infarction, non-fatal stroke, and transient ischemic attacks occur in $3-20 \%$ of patients undergoing CABG according to various authors (Taggart et al., 1999; Bokeria et al., 2008). The impairment of cognitive abilities such as concentration, short-term memory, and speed of information processing occurs in $40-80 \%$ of patients after CABG (Bokeria et al., 2006; Newman, 2007; Stroobant and Vingerhoets, 2008). It was shown that these cognitive deficits are transient and disappear within a few months, but sometimes may persist about 1 year after surgery in 35\% patients (Bokeria et al., 2006). Significant differences in the number of patients with postoperative cognitive dysfunction (POCD) may be associated with using variety of cognitive tests battery, the different criteria to determine cognitive impairment, for patient selection and the evaluation period of patients after surgery (Silbert et al., 2004; Selnes et al., 2006). It was established that elderly patients in the age $\geq 70$ years are high-risk group and have a higher incidence of adverse cerebral outcomes. Currently, there is more evidence that the cognitive impairment in old age is a strong, independent predictor of subsequent mortality (Smits et al., 1999; Bassuk et al., 2000; Pavlik et al., 2003).

The pathogenesis of POCD is multifactorial and in its development contribute to systemic inflammatory response, postoperative pain and sleep disorders, intraoperative factors (Vibha et al., 2006).

Previous studies have shown that the internal carotid artery (ICA) stenosis $>70 \%$ is a risk factor for ischemic brain injury in patients with coronary heart disease (CHD) undergoing CABG (Bokeria et al., 2006). However, the impact of low and moderate stenoses ICA $(<50 \%)$ on the brain functions after CABG are unknown. Meanwhile, the patients with concomitant carotid and coronary artery disease required performing single-stage or phase surgery. The issue of phasing operations - could be selected, in particular, depending on the degree of ICA stenosis.

\section{MATERIALS AND METHODS SUBJECTS}

All patients gave informed consent to participate in a prospective study aimed at studying changes in cognitive function after CABG. The study design was approved by the Ethics Committee of the Institute.

The patients older than 70 years, with initial depressive symptoms identified by the Beck Depression Inventory, dementia [sum 
score of Mini-Mental State Examination (MMSE) $\leq 24$ scores, frontal assessment battery $(\mathrm{FAB}) \leq 11$ scores] were excluded. Also the subjects with known history of rhythm disturbances, heart failure FC NYHA IV, concomitant diseases (chronic obstructive lung disease, cancer pathology), diseases of the central nervous system, any episodes of cerebrovascular accident, brain injuries were excluded. All patients were examined by a neurologist and underwent multispiral computed tomography (CT) in the preoperative period for detecting any abnormalities of the nervous system. To take account of the functional brain asymmetry only right-handed patients were included in the study.

Forty five patients were divided into two groups: with ICA stenosis - GR1 ( $n=20$, mean age $55.1 \pm 4.5$ years $)$ and without one - GR2 ( $n=25$, mean age $56.8 \pm 5.51$ years), the degree of stenosis did not exceed 50\%. Atherosclerotic stenoses in most cases were located in the ostium of ICAs. Before surgery the patients groups were comparable to a history of $\mathrm{CHD}$, the severity of heart failure (FC NYHA), ejection fraction (EF), neurological, and emotional status (see Table 1).

All patients were treated before and after surgery basic and symptomatic therapy according to general principles of treatment of patients with coronary artery disease, congestive heart failure (CHF), and hypertension (National guidelines, 2009, 2008): low-salt $(<1 \mathrm{~g} /$ day $)$ and hypocholesterolic diet, beta-blockers (bisoprolol fumarate), angiotensin-converting enzyme inhibitors (enalapril maleate), statins (rosuvastatin). Anesthesia and perfusion was carried out by the standard procedure: a combined endotracheal anesthesia (Diprivan, Fentanyl, Sevoflurane). For all patients CABG surgery was performed planned with normothermia. Three arteries were operated - in $42 \%$ of patients, $2-48 \%, 1-10 \%$. The average number of grafts was $2.8 \pm 0.2$. In eight cases resection of the left ventricle aneurysm was made.

Table 1 | Demographical and clinical characteristics of the groups.

\begin{tabular}{lccc}
\hline & GR1 $\boldsymbol{n}=\mathbf{2 0}$ & GR2 $\boldsymbol{n}=\mathbf{2 5}$ & $\boldsymbol{p}$ \\
\hline Age (years) & $55.1 \pm 5.3$ & $56.8 \pm 5.51$ & $>0.05$ \\
History of CHD (years) & $4.6 \pm 1.6$ & $4.7 \pm 1.3$ & $>0.05$ \\
CCS & & & \\
II & $70 \%$ & $63 \%$ & $>0.05$ \\
III & $30 \%$ & $37 \%$ & \\
FC NYHA & & & \\
II & $75 \%$ & $68 \%$ & $>0.05$ \\
III & $25 \%$ & $32 \%$ & \\
EF\% & $53.17 \pm 9.6$ & $54.6 \pm 5.2$ & $>0.05$ \\
MMSE (scores) & $25.8 \pm 1.74$ & $25.6 \pm 1.66$ & $>0.05$ \\
FAB (scores) & $16.2 \pm 1.62$ & $16.6 \pm 0.9$ & $>0.05$ \\
Beck (scores) & $2.1 \pm 2$ & $2.7 \pm 1.1$ & $>0.05$ \\
Spielberger (scores) & $42.02 \pm 1.0$ & $43.0 \pm 0.7$ & $>0.05$ \\
\hline
\end{tabular}

CCS, functional class of angina by Canadian cardiovascular society; FC NYHA, functional class of heart failure by New York Heart Association; EF, ejection fraction; MMSE, Mini-mental state examination; FAB, frontal assessment battery; Beck, Beck depression inventory scale; Spielberger, Spielberger state and trait anxiety questionnaire.
The mean bypass time $(94.6 \pm 20.87$ and $95.3 \pm 15.8 \mathrm{~min})$ and aortic cross-clamping (50.3 \pm 21.85 and $51.9 \pm 20.22 \mathrm{~min})$ did not differ between the patients groups. The invasive hemodynamic monitoring was carried out during an operation; no episodes of hypotension were noted. Also oxygenation of the cerebral cortex (rSO2) in real time ("INVOX-3100," SOMANETICS, USA) was monitored at all surgery stages. A hypoxia of brain was not observed.

\section{PROCEDURE}

All patients underwent neuropsychological testing at 3-5 days before surgery, 7-10 days after surgery, and 1 and 6 months after surgery. Each study subject was tested on dementia using the MMSE and FAB scale, the level of depression was assessed by Beck Depression Inventory scale, state and trait anxiety - Spielberger questionnaire.

\section{NEUROPSYCHOLOGICAL EXAMINATION}

Neuropsychological testing was performed using the automated complex software (Status PF) and included an assessment of the complex visual-motor reaction (CVMR) with evaluation of mean reaction time and the number of errors, short-term memory (the 10 numbers memorizing test), and attention (the Burdon's test).

\section{ELECTROPHYSIOLOGICAL EXAMINATION}

Electroencephalographic (EEG) study was performed in 25 patients GR1 and 11 patients GR2 at three time points: $3-5$ days before surgery, 7-10 days after surgery, and 1 month after surgery. The reason for fewer EEG registrations was the patient's failure in comparison with the data of neuropsychological testing. High-resolution monopolar EEG (62 channels, the bandwidth $0.1-50.0 \mathrm{~Hz}$ ) with closed (EC) and open eyes (EO) was recorded in both groups patients. EEG was amplified by Neuvo electroencephalograph (Compumedics, USA) using a modified 64-channel cap with sintered $\mathrm{Ag} / \mathrm{AgCl}$ electrodes (QuikCap, NeuroSoft Inc., USA). A reference electrode was attached on the tip of the nose, a ground electrode - to the center of the forehead. Electrode resistance was maintained $<20 \mathrm{k} \Omega$. Visual inspection of the eye-movement, myographic, and other artifacts was conducted. Artifact-free EEG fragments were divided into $2 \mathrm{~s}$ epochs and Fourier transformed. For each subject EEG power values were averaged within the delta $(0-4 \mathrm{~Hz})$, teta1 $(4-6 \mathrm{~Hz})$, teta2 $(6-8 \mathrm{~Hz})$, alpha1 $(8-10 \mathrm{~Hz})$, alpha2 $(10-13 \mathrm{~Hz})$, beta1 $(13-20 \mathrm{~Hz})$, beta2 $(20-30 \mathrm{~Hz})$. The mean values of the total EEG power in each band were obtained.

\section{STATISTICAL ANALYSIS}

The statistical software package Statistica 6.0 (Stat. Soft, Inc., 1984-2001) was used for all types of analysis of the variables. The mean and standard deviation was assessed. Wilcoxon and Mann-Whitney tests were used to identify differences in quantitative terms. EEG data (mean values of the total EEG power) were log-transformed to normalize the distribution and statistically analyzed using Student's $t$-test for independent variables. Correlations between EEG and neuropsychological tests were calculated using Pearson's coefficient. 


\section{RESULTS}

As seen in Table 1, GR1 and GR2 were comparable as regards to baseline characteristics, such as age, clinical symptoms, emotional status, and non-specific general level of cognitive ability (measured by the MMSE, FAB scales) before surgery.

\section{NEUROPSYCHOLOGICAL OUTCOMES}

The analyses of baseline neurophysiological parameters revealed non-significant differences between GR1 and GR2, except the number of errors in the CVMR test. After surgery (7-10 days and 6 month), the patients in the GR2 had significantly more errors in the CVMR test than GR1 (see Figure 1).

The Burdon's test showed significant differences between groups at 7-10 days, 1 and 6 months after CABG. The patients of GR2 had less number of processed symbols then GR1 (see Figure 2).

The results of the 10 numbers memorizing test showed less memorized numbers in the GR2 patients at 7-10 days after surgery. The similar changes persisted at 1 and 6 months after surgery, but were non-significant (see Figure 3).

\section{EEG FINDINGS}

The analysis of theta 1 and theta 2 power changes with EC showed no significant differences between groups at baseline (Figure 4). At 7-10 days after CABG, the patients of the GR2 had higher values of teta $1(p=0.06)$ and teta 2 power $(p=0.048)$ in comparison with GR1. At 1 month after surgery there were no significant differences between groups. The differences in theta power with EO between GR1 and GR2 were not found at baseline or at either postoperative time point.

The differences of the alpha 1 and alpha 2 power between GR1 and GR2 were revealed only with EO. Before surgery, the patients of the GR2 showed higher alpha power values compared to GR1 (Figure 5). The differences for alpha 1 rhythm were found at the level of trend, and statistically significant for alpha 2 rhythm. The similar differences between patients groups were found at 7-10 days after CABG. One month after surgery there were no differences of the alpha 1 and alpha 2 power between GR 1 and GR2.

\section{EEG AND NEUROPSYCHOLOGICAL CORRELATIONS}

The correlation analysis showed that before CABG more numbers of errors during the CVMR test was associated with higher values of the beta1 $(R=0.41 ; p=0.04)$ and beta2 $(R=0.39 ; p=0.05)$ power with $\mathrm{EO}$ in all patients. Also negative correlations between the memorized numbers and the delta power with EC and EO $(R=-0.45 ; p=0.02$ and $R=-0.54 ; p=0.005$, respectively) were found.

After CABG, at 7-10 days, negative correlations between attention characteristics and the thetal and 2 power with EC and the alphal and 2 power - with EO were revealed. The higher values of rhythms power were associated with the lower numbers of processed symbols on the third and fourth minute of Burdon's test, and the lower total number of processed symbols (Table 2).

At 1 month after CABG were revealed relationships between the memorized numbers and the theta 2 power with EC and the alpha 1 power with EO. The higher power of these rhythms was correlated with the lower memorized numbers in the all patients ( $R=-0.62 ; p=0.02$ and $R=-0.55 ; p=0.04$, respectively).

Thus, the worst performance of neuropsychological tests was accompanied by the higher theta and alpha rhythms power in the resting EEG at 1 month follow-up.

\section{DISCUSSION}

Previous studies (Alga et al., 2003; Bokeria et al., 2008) have shown that the stenotic lesions of carotid arteries are not only a risk factor for stroke, but may be considered as independent predictors of
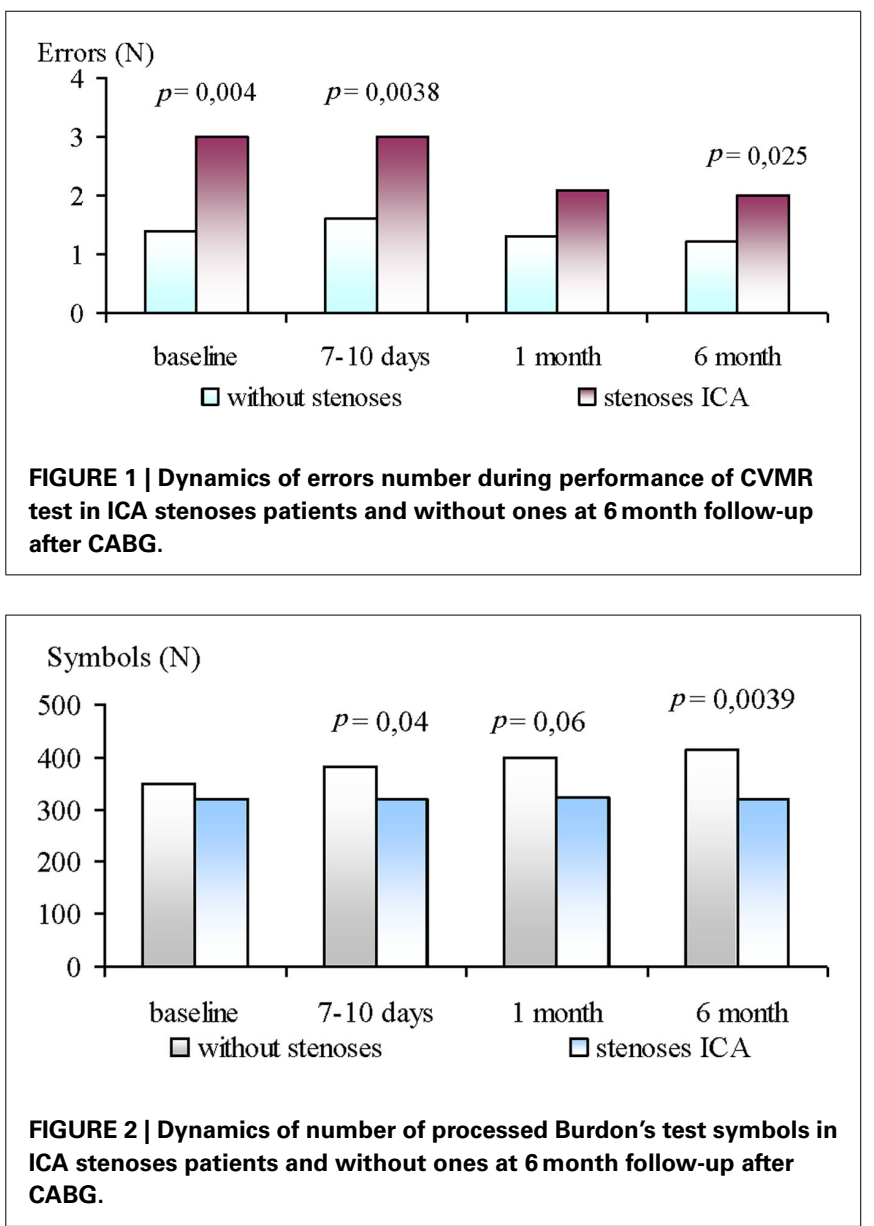

Memorized number (N)

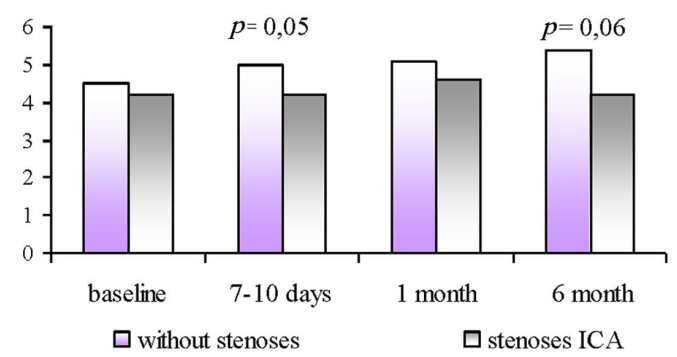

FIGURE 3 | Dynamics of memorized numbers in ICA stenoses patients and without ones at $\mathbf{6}$ month follow-up after CABG. 

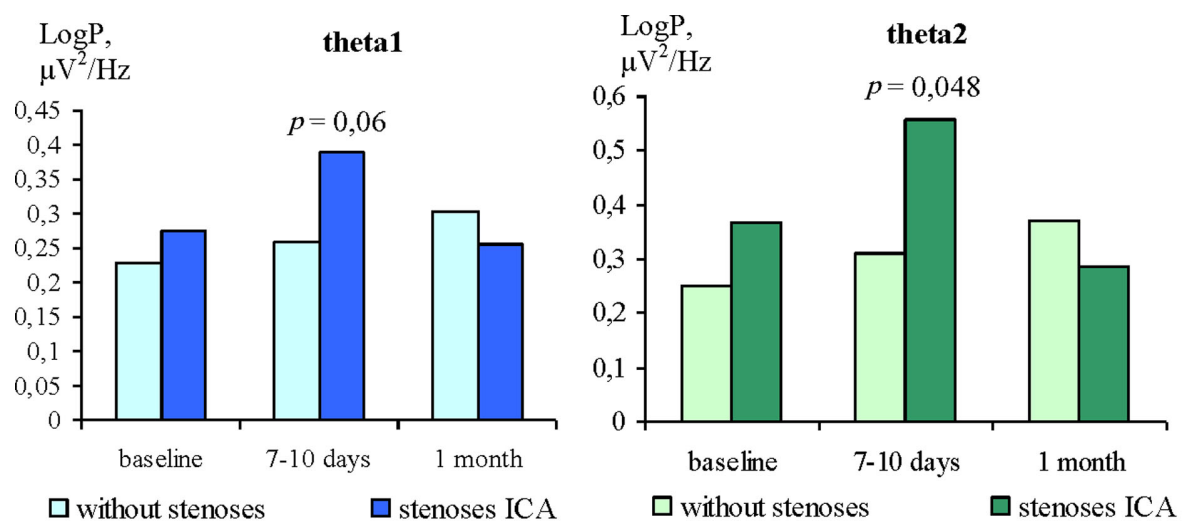

FIGURE 4 | Differences of theta rhythms power with EC in ICA stenoses patients and without ones.
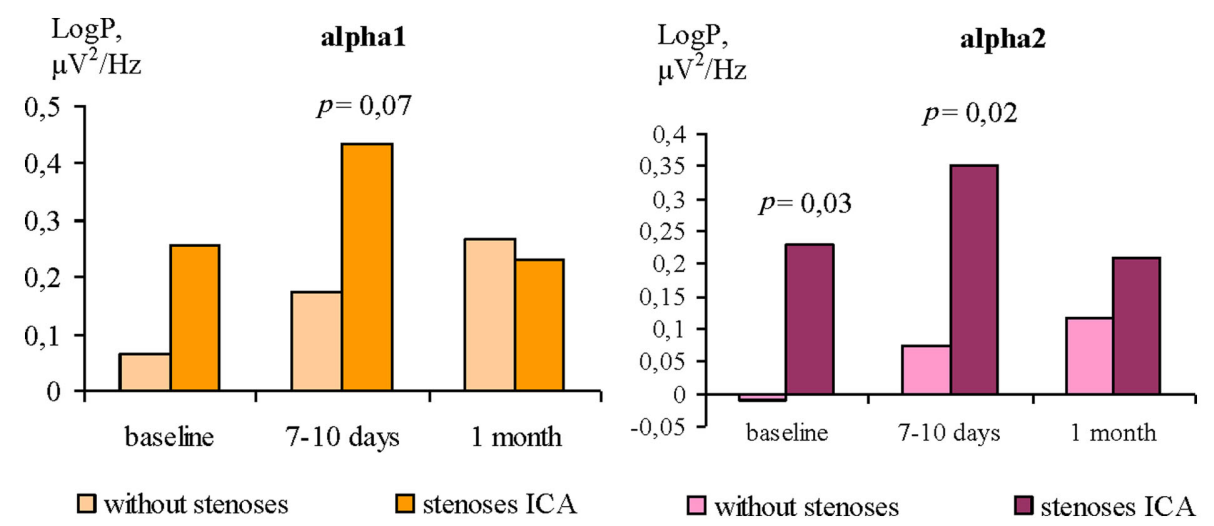

FIGURE 5 | Differences of alpha rhythms power with EO in ICA stenoses patients and without ones.

Table 2 |The correlations between EEG and neuropsychological parameters at 7-10 days after CABG.

\begin{tabular}{llllll}
\hline Burdon's test & $\begin{array}{l}\text { Numbers of processed } \\
\text { symbols on the } \\
\text { first test minute }\end{array}$ & $\begin{array}{l}\text { Numbers of processed } \\
\text { symbols on the } \\
\text { second test minute }\end{array}$ & $\begin{array}{l}\text { Numbers of processed } \\
\text { symbols on the } \\
\text { third test minute }\end{array}$ & $\begin{array}{l}\text { Numbers of processed } \\
\text { symbols on the } \\
\text { fourth test minute }\end{array}$ & $\begin{array}{l}\text { Total number of } \\
\text { processed symbols }\end{array}$ \\
\hline Theta $1 ;(E C)$ & $R=0.14 ; p=0.63$ & $R=0.32 ; p=0.24$ & $R=0.29 ; p=0.29$ & $R=0.63 ; p=0.01$ & $R=0.45 ; p=0.08$ \\
Theta2; $(E C)$ & $R=-0.07 ; p=0.82$ & $R=-0.05 ; p=0.88$ & $R=-0.70 ; p=0.004$ & $R=-0.54 ; p=0.04$ & $R=-0.50 ; p=0.048$ \\
Alpha $1 ;(E O)$ & $R=-0.28 ; p=0.32$ & $R=0.15 ; p=0.60$ & $R=-0.87 ; p=0.0001$ & $R=-0.54 ; p=0.04$ & $R=-0.52 ; p=0.04$ \\
Alpha 2; (EO) & $R=-0.17 ; p=0.55$ & $R=0.06 ; p=0.83$ & $R=-0.75 ; p=0.001$ & $R=-0.57 ; p=0.03$ & $R=-0.49 ; p=0.05$
\end{tabular}

sudden cardiac death. This fact is particularly important for the patients with concomitant carotid and coronary artery disease, who planned for open-heart surgery. CABG is the most effective method of surgical treatment of CHD, improving life quality of the patients and for certain categories of patients increasing lifespan (Akchurin and Shiryaev, 2004; Stroobant and Vingerhoets, 2008). However, the development of postoperative cognitive impairment may reduce the surgery efficiency and may be a reliable marker of adverse long-term prognosis (dementia, death; Newman and Harrison, 2002; Bokeria et al., 2008). Our data suggest that the worst performance of neuropsychological tests was observed in patients with low and moderate ICA stenoses in comparison to patients without one both before and after CABG. We have also found that patients with low and moderate ICA stenoses showed a greater degree of the cerebral cortex inhibition. The higher power of alpha rhythm when patients rested with eyes open was observed in ICA stenosis group in the pre- and post-operative periods. We can assume that the low or moderate carotid artery stenoses concomitant with coronary artery disease contribute to non-optimal level of cortical activation for normal 
processing of incoming information. In turn, this leads to the cognitive impairment. The intensification of cognitive impairment in postoperative CABG patients with low and moderate carotid stenoses may be associated with lower adaptive mechanisms associated with resistance to cerebral ischemia. The reason may be already existing chronic brain ischemia and acute episode of global ischemia during on-pump CABG. This assumption is confirmed the higher theta power values in the resting EEG detected in these patients after CABG. Our data are consistent with the results of Gugino et al. (2004), where it was shown an increase of the theta power under cerebral blood flow below $22 \mathrm{ml} / 100 \mathrm{~g} / \mathrm{min}$. The other studies has reported (Sazonova, 1999; Ogurtsova, 2005) about theta increase during endovascular intervention (temporary occlusion ICA by balloon system). Furthermore, in our study

\section{REFERENCES}

Akchurin, R. S., and Shiryaev, A. A. (2004). Actual Problems of Coronary Surgery. Moscow: GEOTAR-MED.

Alga, A., Gates, P. C., Fox, A. J., Hachinski, V., Barnett, H. J., and North American Symptomatic Carotid Endarterectomy Trial (NASCET) Group. (2003). Side of brain infarction and long-term risk of sudden death in patients with symptomatic carotid disease. Stroke 34, 2871-2875.

Bassuk, S., Wypij, D., and Berkman, L. (2000). Cognitive impairment and mortality in the communitydwelling elderly. Am. J. Epidemiol. 151, 676-688.

Bokeria, L. A., Bukharin, V. A., Rabotnikov, V. S., and Alshibaya, M. D. (2006). Surgical Treatment of Patients with Coronary Artery Disease with Lesions of the Brachiocephalic Arteries, 2nd Edn. revised and updated. M.: NTSSSH them. AN Bakuleva RAMS. 176.

Bokeria, L. A., Kamchatnov, P. R., Klyuchnikov, I. V., Alaverdyan, A. G., and Gusev, E. I. (2008). Cerebrovascular disorders in patients with coronary bypass surgery. J. Neurol. Psychiatry 3, 90-94.

Gugino, L. D., Aglio, L. S., and Yli-Hankala, A. (2004). Monitoring the electroencephalogram during bypass procedures. Semin. Cardiothorac. Vasc. Anesth. 8, 61-83.
Newman, M. (2007). Open heart surgery and cognitive decline. Cleve. Clin. J. Med. 74, 52-55.

Newman, S. P., and Harrison, M. J. (2002). Coronary-artery bypass surgery and the brain: persisting concerns. Lancet Neurol. 1, 119-125.

Ogurtsova, A. A. (2005). Electrophysiological Monitoring During Operations on the Brain Vessels. Ph.D. thesis, Research Institute of Neurosurgery N. N. Burdenko, Russian Academy of Medical Sciences, Moscow.

Pavlik, V. N., Alves de Moraes, S., Szklo, M., Knopman, D. S., Mosley, T. H., and Hyman, D. J. (2003). Relation between cognitive function and mortality in middle-aged adults: the atherosclerosis risk in communities study. Am. J. Epidemiol. 157, 327-334.

Sazonova, O. B. (1999). Monitoring of spontaneous bioelectric brain activity in neuroanesthesiology and neurosurgery. Grew up. Zh. An. IT 1, 64-70.

Selnes, O. A., Pham, L., Zeger, S., and McKhann, G. M. (2006). Defining cognitive change after CABG: decline versus normal variability. Ann. Thorac. Surg. 82, 388-390.

Silbert, B. S., Maruff, P., Evered, L. A., Scott, D. A., Kalpokas, M, Martin, K. J, Lewis, M. S., and Myles, P. S. (2004). Detection of cognitive

we obtained the correlation between the decline in attention and memory and low-frequency EEG activity in the rest after CABG. This fact also supports the hypothesis of ischemic brain injury and developing cognitive impairment during open-heart surgery.

\section{CONCLUSION}

Thus, patients with low and moderate ICA stenoses have worse dynamics of neurophysiological status in comparison to patients without stenotic lesions of carotid arteries at 6 month follow-up after on-pump coronary artery bypass surgery. The results of our study suggest that the presence even $\leq 50 \%$ carotid artery stenosis is a risk factor for brain ischemia and cognitive impairment in patients undergoing on-pump CABG.

decline after coronary surgery: a comparison of computerized and conventional tests. Br. J. Anaesth. 92, 814-820.

Smits, C., Deeg, D., Kriegsman, D. and Schmand, B. (1999). Cognitive functioning and health as determinants of mortality in an older population. Am. J. Epidemiol. 150, 978-986.

Stroobant, N., and Vingerhoets, G. (2008). Depression, anxiety, and neuropsychological performance in coronary artery bypass graft patients: a follow-up study. Psychosomatics 49, 326-331.

Taggart, D. P., Browne, S. M., Halligan, P. W., and Wade, D. T. (1999). Is cardiopulmonary bypass still the cause of cognitive dysfunction after cardiac operations? J. Thorac. Cardiovasc. Surg. 118, 414-420.

Vibha, D., Kalita, J., Agarwal, R., and Misra, U. K. (2006). Coronary artery bypass surgery: does it lead to cognitive impairment? Ann. Indian Acad. Neurol. 9, 145-151.

Conflict of Interest Statement: In the present study, there are no any actual or potential conflicts including any financial, personal, or other relationships with other people or organizations. Author's institution has no contracts relating to this research through which it or any other organization may stand to gain financially now or in the future.
Authors or their institutions have no a financial interest in this work. This manuscript has not been published elsewhere and is not under review with another journal. The present investigation adhered to the tenets of the Declaration of Helsinki and was approved by the institutional human experimentation committee. Informed consent was obtained from all subjects after the nature of the procedure had been explained. All authors have reviewed the contents of the manuscript being submitted, approve of its contents, and validate the accuracy of the data.

Received: 14 December 2011; accepted: 02 January 2012; published online: 18 January 2012.

Citation: Trubnikova O, Tarasova I and Barbarash $O$ (2012) The influence of low and moderate carotid stenosis on neurophysiologic status of patients undergoing on-pump coronary artery bypass grafting. Front. Neur. 3:1. doi: 10.3389/fneur.2012.00001

This article was submitted to Frontiers in Dementia, a specialty of Frontiers in Neurology.

Copyright (C) 2012 Trubnikova, Tarasova and Barbarash. This is an open-access article distributed under the terms of the Creative Commons Attribution Non Commercial License, which permits noncommercial use, distribution, and reproduction in other forums, provided the original authors and source are credited. 